\title{
ÉTUDE DU COMPORTEMENT DU SAUMON ATLANTIQUE (SALMO SALAR L.) AU NIVEAU DE AMÉNAGEMENT HYDROÉLECTRIQUE DE BAIGTS (GAVE DE PAU) LORS DE SA MIGRATION ANADROME.
}

\author{
M. CHANSEAU et M. LARINIER
}

C.JP-CEMAGREF-GHAAPPE, Institut de Mécanique des Fluides, Avenue du Professeur Camille Soula, 31400 Toulouse, France.

Ri ̧u le 07 mai 1999

A. septé le 19 juillet 1999

Received 07 May, 1999

Accepted 19 July, 1999

\section{Ris SUMÉ}

Le comportement de 19 saumons adultes (Salmo salar L.) a été étudié au niveau de l'anénagement hydroélectrique de Baigts à l'aide de la technique de radiotélémétrie lors de trois expérimentations menées en été et en automne de 1995 à 1997.

La majorité des poissons a stationné bien à l'aval de l'obstacle au cours de leur blocage. Les durées de présence en continu sur le site et les départs à l'aval étaient généralement inférieurs à 1 heure, les poissons se repliant au maximum à 200-300 m à l'aval de l'ouvrage avant de se présenter de nouveau sur le site. Lors de replis de durées plus importantes, généralement supérieures à 6 heures, les poissons stationnaient principalement dans un profond s'étendant de $500 \mathrm{~m}$ à 1,2 kilomètre de l'obstacle. Les départs du site se produisaient plutôt au crépuscule et les retours au pied de l'obstacle préférentiellement à l'aube, révélant une rythmicité comportementale des poissons au cours du nycthémère.

Les températures moyennes journalières de l'eau et les débits turbinés au niveau de Pusine ont eu une influence sur les présences des poissons au niveau de l'obstacle. Les saumons se présentaient plus souvent mais stationnaient moins longuement au pied de l'aménagement lorsque les températures étaient supérieures à $16^{\circ} \mathrm{C}$. Les présences des poissons dans le canal de fuite étaient nettement réduites pour des températures supérieures à $20^{\circ} \mathrm{C}$ tandis que le stationnement au pied du barrage était plus important pour un fonctionnement réduit (débits turbinés inférieurs à $50 \mathrm{~m} 3 / \mathrm{s}$ ) de l'usine.

Les études ont mis en évidence l'impact important de l'aménagement sur la migration anadrome des saumons, en regard notamment des durées de blocage importantes induites par l'aménagement (jusqu'à 137 jours) et du faible nombre de poissons (34\%) qui a réussi à passer à l'amont. L'emplacement de l'entrée du dispositif de franchissement, situé pourtant à l'opposé du canal de fuite, ne peut être tenu pour seul responsable, tous les poissons s'étant présentés de nombreuses fois au pied de la passe à poissons. Les conditions rencontrées par les poissons au niveau de l'entrée de la passe à poissons n'ont pas paru optimales, les saumons stationnant le plus souvent moins de 5 minutes devant l'entrée du dispositif de franchissement lors de chaque incursion. De plus, les poissons ont eu des réticences à s'engager à l'intérieur du dispositif, 0,86\% seulement des incursions au niveau de la passe à poissons étant suivies d'une entrée dans le dispositif.

Mots-clés : migration anadrome, aménagement hydroélectrique, radiotélémétrie, saumon atlantique, comportement. 


\section{THE BEHAVIOUR OF RETURNING ADULT ATLANTIC SALMON (SALMO SALAR L: IN THE VICINITY OF BAIGTS HYDROELECTRIC POWER PLANT ON THE PAU RIVE AS DETERMINED BY RADIOTELEMETRY.}

\section{ABSTRACT}

The behaviour of 19 radiotagged adult Atlantic salmon (Salmo salar L.) was studit. during the summer and autumn of three successive years (1995 to 1997) in the vicinity:) the Baigts hydroelectric plant.

Most of the time, the fish stayed away from the dam. Visits to the site and the tin: which they remained downstream were generally short lived (less than 1 hour), with t:? fish generally being within $300 \mathrm{~m}$ downstream from the plant. When fish were absent for: longer time, i.e. more than 6 hours, they were often observed in a pool located betweet $500 \mathrm{~m}$ and 1.2 kilometer downstream from the obstacle. Behavioural rhythmicity of fisl was pointed out : they approached the plant mainly at dawn and were more present an: active during the day.

Mean daily temperature and turbine discharge influenced salmon behaviour in thi vicinity of the plant. Fish were more often found approaching the plant when the temperature was higher than $16^{\circ} \mathrm{C}$ but stayed less time in the vicinity of the obstacle Presence of fish in the tailrace and at the dam was influenced by temperature and $b$ operation of the hydroelectric power plant with fish being less present in the tailrace wher temperature exceeded $20^{\circ} \mathrm{C}$ and more present at the dam when the turbine discharge wa lower than $50 \mathrm{~m}^{3} / \mathrm{s}$.

Studies revealed that the plant impeded the upstream migration of salmon with fis being delayed up to 137 days and only a small percentage (34\%) being able to pass ove the obstacle. The location of the fish pass, at the opposite side of the tailrace, was nc responsible for its poor efficiency since all fish were monitored near the entrance. Th. conditions encountered by fish at the entrance of the fish pass were not optimal with mos of the visits lasting less than 5 minutes. Moreover, only $0.86 \%$ of the visits resulted in fis moving into the pass.

Key-words : upstream migration, hydroelectric power plant, radiotelemetry, Atlanti salmon, behaviour.

\section{INTRODUCTION}

Dans le cadre d'un suivi par radiotélémétrie de la migration anadrome du saumo atlantique sur le gave de Pau afin de déterminer l'impact des différents obstacles érigé sur l'axe (CHANSEAU, CROZE et LARINIER, 1999), une étude a été effectuée au nivea de l'aménagement de Baigts en été et en automne, de 1995 à 1997.

En plus de l'évaluation per se de l'impact de l'ouvrage, un suivi détaillé de déplacements des saumons au niveau de l'aménagement, et notamment au niveau d l'entrée du dispositif de franchissement, a été réalisé lors des trois année d'expérimentation en regard des risques potentiels de blocage que cet obstacle pouva 
e'gendrer et de sa position très aval sur l'axe de migration. L'étude des stratégies comportementales mises en oeuvre par les poissons au niveau de l'obstacle devait p. imettre de révéler les raisons des éventuelles difficultés de franchissement rencontrées.

Dans un cadre plus général, ces études constituent une approche dans la c. npréhension du comportement des poissons au niveau d'un obstacle, paramètre très ir oortant à prendre en compte afin de faciliter le passage des migrateurs (KYNARD, 1.31). De meilleures informations sont nécessaires dans ce domaine, notamment en ce q. concerne les mécanismes comportementaux responsables de l'attraction des poissons $d$ is les dispositifs de franchissement (NORTHCOTE, 1998).

\section{N. TÉRIEL ET MÉTHODES}

\section{Site d'étude}

L'aménagement hydroélectrique de Baigts (Figure 1) est situé sur le gave de Pau, à er iron 25 kilomètres de la confluence avec le gave d'Oloron et à une cinquantaine de kilsmètres de l'estuaire de l'Adour. Au niveau de l'aménagement de Baigts (superficie du be $3 \sin$ versant : $2575 \mathrm{~km}^{2}$ ), le module interannuel du gave de Pau, calculé sur la période de référence 1973-1997, est de $79 \mathrm{~m}^{3} / \mathrm{s}$. Le régime du cours d'eau est de type nivo-pluvial marqué par une période d'étiage en fin d'été (août et septembre), d'eaux de fonte d'avril à juin et de fortes précipitations pluviales en décembre.

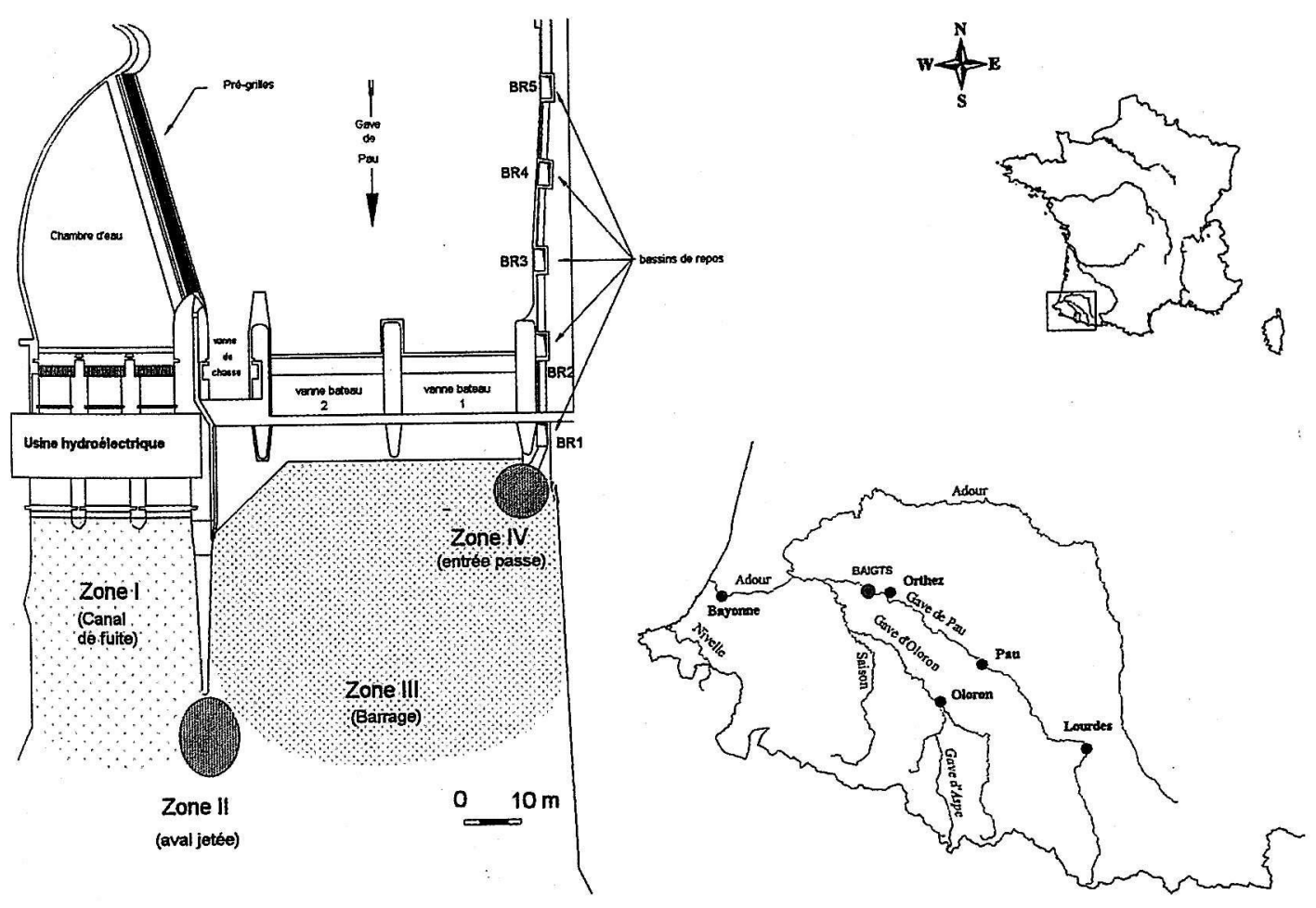

\section{Figure 1}

Situation géographique de l'aménagement hydroélectrique de Baigts.

\section{Figure 1}

Geographical situation of the Baigts hydroelectric power plant. 

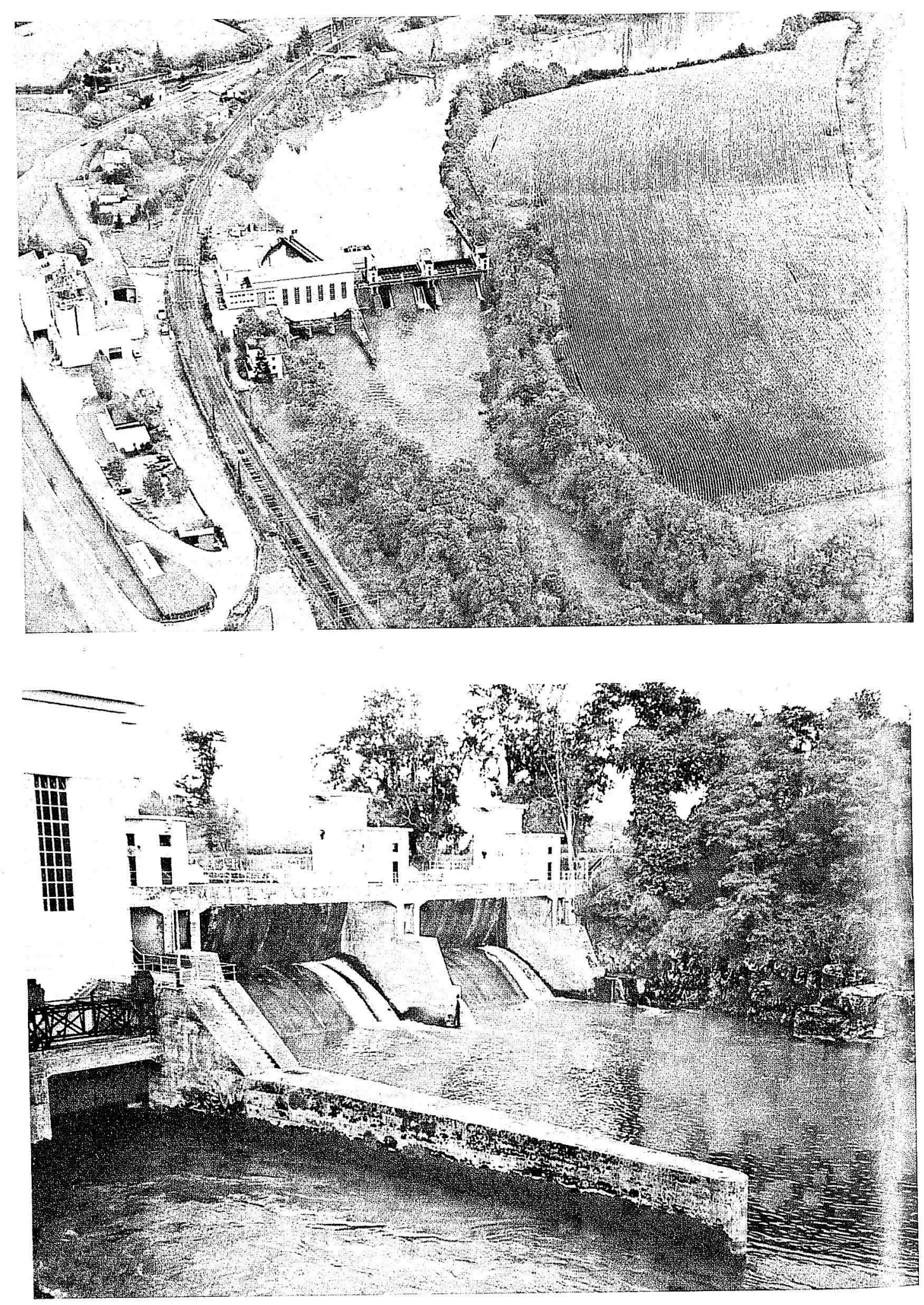

Figure 2

Vue générale de l'aménagement de Baigts et de l'entrée de la passe à poissons.

Figure 2

A general view of the Baigts power plant and of the entrance of the fish pass. 
L'aménagement se compose en rive gauche d'un barrage mobile d'une hauteur de $15.4 \mathrm{~m}$ et d'une longueur de crête de $57 \mathrm{~m}$ équipé de 2 vannes bateaux automatiques (c: pacité d'évacuation : $1200 \mathrm{~m}^{3 / \mathrm{s}}$ ) et d'une vanne de chasse pourvue d'un clapet de st tace (capacité d'évacuation : $300 \mathrm{~m} 3 / \mathrm{s}$ ). En rive droite se trouve l'usine hydroélectrique ql: fonctionne au fil de l'eau. Elle est équipée de 3 turbines Kaplan verticales turbinant cl : cune $30 \mathrm{~m}^{3} / \mathrm{s}$ sous environ $11 \mathrm{~m}$ de chute. Un canal de fuite de $52 \mathrm{~m}$ de long permet d'. 'acuer le débit turbiné (Figure 2).

La passe à poissons, construite en 1941, est située en rive gauche, au niveau du be age. Elle est longue de $82 \mathrm{~m}$ pour un dénivelé de près de $11 \mathrm{~m}$. Elle est composée de 6 lées de pente variant de $16 \%$ à $20 \%$ séparées par 5 bassins de repos. Quatre volées sc équipées de ralentisseurs plans en béton ou métalliques, une de ralentisseurs à ch urons épais en bois et une enfin est constituée de 3 bassins successifs à parois dé ersantes (Figure 3). Le débit dans la passe à poissons est d'environ 500 L/s et un débit co iplémentaire d'attrait de $500 \mathrm{~L} / \mathrm{s}$ transitant par une conduite est injecté au pied du dis ositif de franchissement.

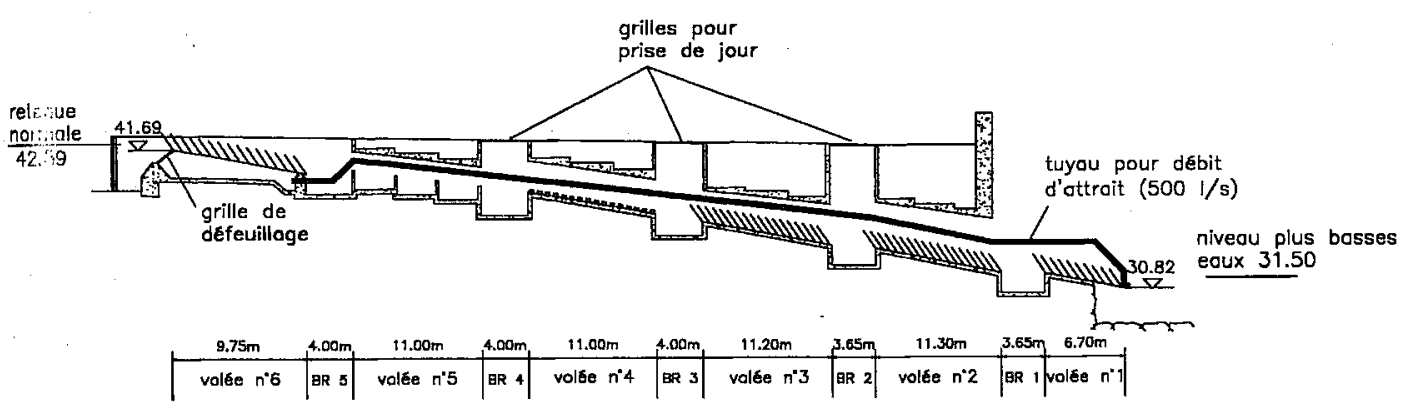

Figure 3

Le dispositif de franchissement de Baigts.

Figure 3

The Baigts fish pass.

\section{Période d'étude}

Le suivi du comportement du saumon atlantique au niveau de l'aménagement de Baigts a été effectué durant trois années consécutives, de 1995 à 1997. Les expérimentations se sont déroulées chaque année de fin juin jusqu'à mi-décembre. En 1997, des travaux au niveau des vannes bateaux du barrage ont été réalisés, nécessitant un abaissement du plan d'eau amont et par conséquent un arrêt de l'usine, du 14 octobre au 17 décembre. Les données recueillies en 1997 après le 14 octobre ne seront pas intégrées dans les analyses ultérieures.

\section{Paramètres du milieu}

La température de l'eau et les débits à l'aval immédiat de l'aménagement ont été relevés tout au long des trois années d'étude. L'enregistrement du fonctionnement des turbines de l'usine et les valeurs de débit à l'aval de l'obstacle ont permis d'évaluer le débit transitant par l'aménagement, le débit turbiné ainsi que le débit évacué par les vannes du barrage. Les 4 périodes du nycthémère (aube, jour, crépuscule et nuit) ont été déterminées sur la base des heures légales de lever et de coucher du soleil. L'aube, d'une durée de 2 heures, correspond à la période comprise entre les heures précédant et suivant 
le lever du soleil. Le crépuscule est la période équivalente pour le coucher du soleil. $C::$ trois paramètres, température, débit et lumière, sont généralement considérés comr: : ceux exerçant les effets les plus marqués sur la migration anadrome du saumon atlantig:at (SMITH, 1985 ; JENSEN, HEGGBERGET et JOHNSEN, 1986 ; POTTER, 1988 JENSEN, JOHNSEN et HANSEN, 1989 ; JONSSON, 1991 ; SMITH et SMITH, 199: JENSEN, HVIDSTEN et JOHNSEN, 1998).

\section{Capture, marquage et technique de radịotélémétrie}

Les saumons ont été capturés dans la passe à poissons de Puyoo, situ i 8 kilomètres à l'aval du site d'étude, et marqués selon la même procédure que celle décrì dans CHANSEAU, CROZE et LARINIER (1999).

Le matériel de radiotélémétrie utilisé (émetteurs, récepteurs, stations réceptric fixes automatiques) est de marque ATS (Advanced Telemetry System).

Au niveau de l'aménagement de Baigts, un certain nombre de stations fixes (jusqu': 7 stations en 1997) composées d'un récepteur relié à un enregistreur numérique (229 Ko a été installé et a permis de délimiter différentes zones de réception. La présence de poissons au pied de l'aménagement a été ainsi suivie en continu tout au long des études Lors des trois années d'expérimentation, 4 zones de réception identiques (Figure 1) or été individualisées de façon à pouvoir comparer les déplacements des saumon radiomarqués.

En 1995, une antenne réceptrice immergée a été installée au niveau de l'entrée d la passe à poissons. En 1996, outre l'entrée du dispositif, les premier et dernier bassins d repos ont été équipés. En 1997, une antenne a été installée dans chacun des 5 bassins d repos.

A l'aval de l'aménagement, la position des saumons était repérée à l'aide d'un antenne omnidirectionnelle fixée sur une voiture. Une localisation plus précise de saumons était effectuée à pied à l'aide d'un récepteur portable et d'une antenn bidirectionnelle.

\section{RÉSULTATS}

Sur les trois années d'étude, 43 saumons ont été marqués et lâchés à l'amor immédiat du barrage de Puyoo. Trente-deux poissons se sont présentés au pied d l'aménagement de Baigts. En ce qui concerne les 11 poissons qui ne sont pas parvenu jusqu'à l'obstacle, un individu a régurgité son émetteur dans les heures suivant sa remis à l'eau et 9 autres ont disparu peu de temps après le lâcher (arrêt de l'émetteur o dévalaison). Un poisson enfin, marqué et relâché fin juillet à Puyoo, a dévalé et a ét retrouvé au niveau de la confluence des gaves de Pau et d'Oloron où il a stationné plus d 15 jours. Il est ensuite retourné en mer et a été piégé fin novembre sur la Nivelle, a niveau de la station de contrôle des poissons migrateurs située au barrage d'Uxondoa.

\section{Délais entre le lâcher et l'arrivée au pied de l'aménagement}

Les délais entre les lâchers et les arrivées des poissons au niveau de l'obstacle d Baigts ont varié de 8 heures à près de 49 jours, la médiane étant égale à 38 heures Dix poissons ont mis moins de 14 heures, 12 entre 18 heures et 3 jours, 7 entre $3 t$ 15 jours et 3 enfin entre 18 et 49 jours.

Ces délais ne peuvent servir à calculer une vitesse moyenne de migration dans I mesure où ils prennent en compte le temps de récupération des poissons à l'anesthésie $\epsilon$ aux différentes manipulations. A titre indicatif, les vitesses moyennes minimales d déplacement des dix poissons les plus rapides sont comprises entre $0,54 \mathrm{~km} / \mathrm{h}$ et $1 \mathrm{~km} / \mathrm{h}$ 


\section{Impact de l'aménagement : franchissements et durées de blocage}

Onze seulement des 32 poissons (soit $34 \%$ ) qui se sont présentés au niveau de l'c istacle ont réussi à passer à l'amont. Ce pourcentage est resté du même ordre de gr ndeur chaque année (entre $30 \%$ et $40 \%$ pour les trois années d'étude).

Les durées de blocage, calculées en faisant la différence entre l'heure de la pr mière arrivée d'un poisson sur le site et celle de sa dernière réception au pied de l'c stacle, ont varié de 9 heures à plus de 137 jours. En ce qui concerne les 11 saumons ql ont réussi à franchir l'aménagement, ces durées sont comprises entre 9 heures et plus dc. 79 jours, deux poissons ayant été retardés moins de 72 heures, la médiane étant égale à 1 jours. Les poissons qui n'ont pas réussi à passer à l'amont ont été bloqués entre 11 ef : 37 jours, 6 d'entre eux l'ayant été plus de 60 jours. Quatre poissons ont disparu peu de te ps après leur arrivée sur le site et ont été retrouvés, lors de différentes re onnaissances aériennes ou en voiture, sur le bassin du gave d'Oloron.

Dans toutes les études de télémétrie, il faut supposer que les émetteurs n'interfèrent pe: avec la survie, les performances ou le comportement des poissons (BARAS et LAAARDERE, 1995). Seuls 19 des 32 poissons qui se sont présentés au pied de l'anénagement ont été retenus, dont les 11 saumons qui sont parvenus à franchir l'onstacle (Tableau 1). Ont été écartés des analyses ultérieures, outre un individu qui a régurgité son émetteur dans les heures suivant son arrivée sur le site d'étude, les poissons qui n'ont été reçus que très peu de temps au pied de l'aménagement.

\section{Tableau I}

Détails des 19 saumons radiomarqués suivis au cours des 3 années d'étude.

\section{Table I}

\section{Details of 19 radiotagged salmon tracked during the 3-year study.}

\begin{tabular}{|c|c|c|c|c|c|c|}
\hline $\begin{array}{c}\text { POISSONS } \\
\text { (fréquence de } \\
\text { l'émetteur -Mhz-) }\end{array}$ & $\begin{array}{l}\text { LONGUEUR } \\
\text { TOTALE } \\
(e n \mathrm{~cm})\end{array}$ & SEXE & $\begin{array}{c}\text { DATE } \\
\text { DU } \\
\text { LACHER }\end{array}$ & $\begin{array}{l}\text { DELAI } \\
\text { LACHER } \\
\text { ARRIVEE }\end{array}$ & BLOCAGE & $\begin{array}{c}\text { PASSAGE } \\
\text { AMONT } \\
\text { BAIGTS }\end{array}$ \\
\hline 49,660 & 68,5 & $\mathrm{~F}$ & $07 / 07 / 95$ & $13 \mathrm{~h}$ & 137 jours & NON \\
\hline 49,680 & 69 & $\mathrm{~F}$ & 28/06/95 & $20 \mathrm{~h}$ & 10 jours & OUI \\
\hline 49,740 & 68,2 & M & 05/10/95 & $11 \mathrm{j} 17 \mathrm{~h}$ & 32 jours & OUI \\
\hline 49,760 & 68 & $F$ & $01 / 07 / 95$ & $8 \mathrm{~h}$ & 36 jours & NON \\
\hline $49,860 \mathrm{~A}$ & 67,2 & $\mathrm{~F}$ & 28/06/95 & $2 \mathrm{j} 07 \mathrm{~h}$ & 11 jours & NON \\
\hline $49,860 \mathrm{~B}$ & 69,8 & M & 05/09/95 & $18 \mathrm{~h}$ & 85 jours & NON \\
\hline 48,721 & 66,5 & $M$ & $21 / 08 / 96$ & $27 \mathrm{~h}$ & 21 jours & NON \\
\hline 48,741 & 67,7 & $M$ & $23 / 08 / 96$ & $24 j$ & 21 jours & OUI \\
\hline 48,781 & 70,6 & $\mathrm{~F}$ & 05/09/96 & $11 \mathrm{j}$ & 11 jours & NON \\
\hline 48,801 & 69,9 & $M$ & 05/09/96 & $13 \mathrm{~h}$ & 61 jours & OUI \\
\hline 48,841 & 65,3 & $M$ & $11 / 09 / 96$ & $5 \mathrm{j} 15 \mathrm{~h}$ & 18 jours & OUI \\
\hline 48,881 & 69,4 & $\mathrm{~F}$ & $17 / 09 / 96$ & $8 h$ & 14 jours & OUI \\
\hline 48,140 & 70,6 & $F ?$ & 07/07/97 & $1 \mathrm{j} 09 \mathrm{~h}$ & 97 jours & NON \\
\hline 48,180 & 66,8 & $M$ & $09 / 07 / 97$ & $10 \mathrm{~h}$ & 97 jours & NON \\
\hline 48,200 & 67 & $M$ & 08/07/97 & $1 \mathrm{j} 21 \mathrm{~h}$ & 77 jours & OUI \\
\hline 48,220 & 63,4 & M? & $10 / 07 / 97$ & $8 h$ & 17 jours & OUI \\
\hline 48,280 & 73,8 & $M$ & $11 / 07 / 97$ & $2 \mathrm{j} 16 \mathrm{~h}$ & 79 jours & OUI \\
\hline 48,480 & 67,9 & $F$ & $28 / 07 / 97$ & $2 \mathrm{j} 01 \mathrm{~h}$ & 9 heures & OUI \\
\hline 48,620 & 69,8 & M? & 07/08/97 & $6 \mathrm{j} 07 \mathrm{~h}$ & 3 jours & OUI \\
\hline
\end{tabular}




\section{Présence des poissons au pied de l'obstacle}

Durées de présence et caractéristiques des incursions au pied de l'aménagemen.

La grande majorité des saumons (13 sur 19 , soit $68,4 \%$ ) a stationné, au cours blocage, non pas au pied de l'obstacle mais à l'aval de l'aménagement, le plus souv: dans un profond situé entre $500 \mathrm{~m}$ et 1,2 kilomètre de l'ouvrage. Deux des 6 poissons a ont passé proportionnellement plus de temps au pied de l'obstacle sont les saumons i ont franchi l'ouvrage le plus rapidement, en moins de 72 heures.

Les incursions au pied de l'aménagement ont été généralement de courte duré $71,5 \%$ (969 sur 1356 ) d'entre elles ne dépassent pas 3 heures ; 3,3\% seulement $c$; présences sur le site sont supérieures à 24 heures. La majorité des replis à l'aval $(855$ 1392 , soit $61,4 \%$ ) n'a pas excédé 3 heures, les poissons demeurant alors généraleme: à proximité immédiate de l'obstacle (200 à $300 \mathrm{~m}$ à l'aval), principalement en rive gauci: en limite de courant. Lors des replis de plus longue durée, généralement supérieure 6 heures, les poissons étaient localisés le plus souvent dans le profond mentionné pi. haut. Seuls $5,1 \%$ des replis à l'aval du site ont eu des durées supérieures à 24 heur: (Figure 4). Les poissons, s'ils n'ont pas passé la plus grande partie de leur temps blocage au pied même de l'obstacle, ne se sont éloignés toutefois que rarement $d$ l'aménagement. Seuls 11 replis d'une distance supérieure à 4 kilomètres ont pu être m en évidence, 7 d'entre eux se produisant durant les mois d'octobre et de novembre. Dei poissons ont ainsi dévalé le seuil de Puyoo situé 8 kilomètres à l'aval, un individu ayal même été localisé à 16 kilomètres à l'aval de Baigts. Ces deux saumons ont par la suit franchi l'obstacle de Puyoo avant de se présenter de nouveau au pied de l'aménagemel de Baigts.
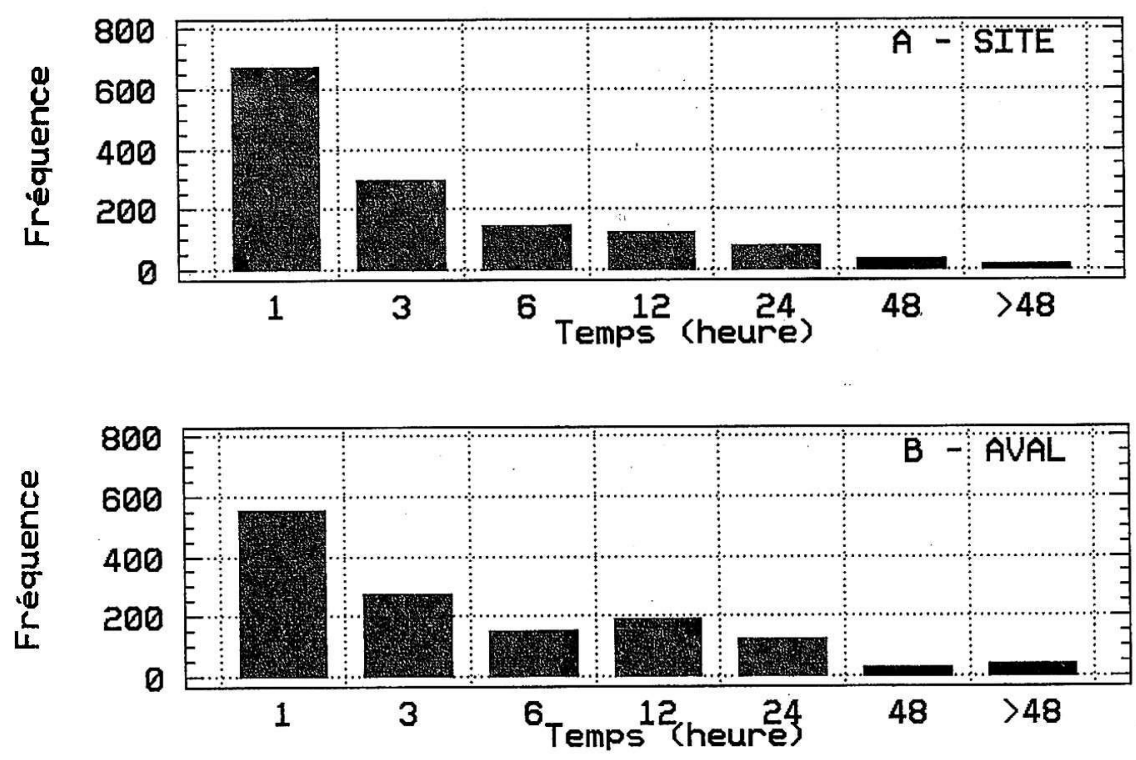

Figure 4A

Durées moyennes de présence des poissons au pied de l'obstacle.

Figure 4B

Durées moyennes de présence des poissons à l'aval de l'aménagement.

Figure 4A

Mean presence duration of fish in the vicinity of the obstacle.

Figure 4B

Mean presence duration of fish further downstream from the plant. 
Le nombre d'incursions sur le site au cours d'une journée varie de 1 à 11 (Figure 5A), les nombres les plus fréquents étant de 1 et 2 . Les temps totaux passés sur le site au cours d'une journée sont très variables et leur distribution très étalée, allant de nioins d'une heure à 24 heures (Figure $5 \mathrm{~B}$ ). Les temps moyens des incursions des pissons au pied de l'obstacle sont faibles, la majorité étant inférieure à 2 heures (i gure 5C).
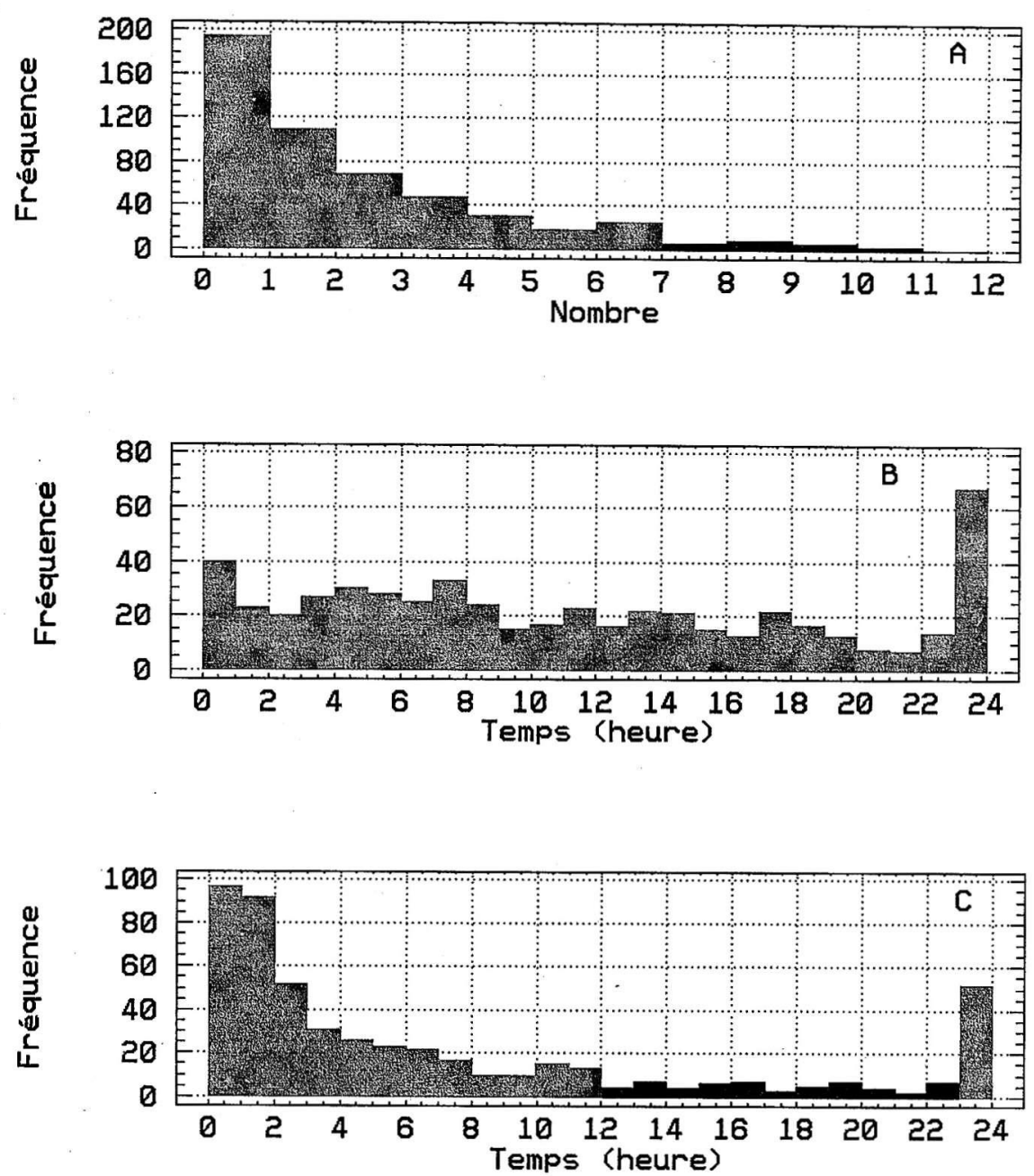

Figure 5A

Nombre d'incursions par jour au pied de l'obstacle.

Figure 5B

Distribution des durées des incursions au pied de l'obstacle.

Figure 5C

Durées moyennes des incursions au pied de l'obstacle.

Figure 5A

Number of approaches per day to the obstacle.

Figure 5B

Distribution of the duration of approaches to the obstacle.

Figure 5C

Mean duration of approaches to the obstacle. 


\section{Présence au cours du nycthémère}

La présence des saumons au pied de l'obstacle a été étudiée au cours des quat's périodes du nycthémère : l'aube, le jour, le crépuscule et la nuit.

Treize poissons sur 19 ont séjourné plus de $50 \%$ de leur temps de présence sur: site en pleine journée. En ce qui concerne les 6 autres saumons, deux individus $c$ séjourné plus de temps au pied de l'obstacle de jour, deux des temps équivalents de jc. et de nuit et deux enfin préférentiellement de nuit. Cependant, si l'on compare les duré: de présence des poissons de nuit avec la somme des durées à l'aube, de jour et : crépuscule, il apparaît qu'aucun saumon n'a stationné majoritairement de nuit au pied l'aménagement. La répartition des durées de présence des saumons au niveau l'aménagement au cours du nycthémère n'est pas aléatoire $\left(\chi^{2}=253 ; p<0,001\right)$. Le te: de contraste met en évidence d'une part que les saumons stationnent préférentielleme , de jour au pied de l'aménagement et d'autre part qu'ils sont moins présents de nt:i (Figure 6A). Pour illustrer ce résultat, on a présenté sur la Figure 6B les pourcentages : présence au cours de chaque période du nycthémère rapportés aux différentes durées 6 ces périodes ( 2 heures pour l'aube et le crépuscule et respectivement 11 heures? 9 heures en moyenne pour le jour et la nuit).
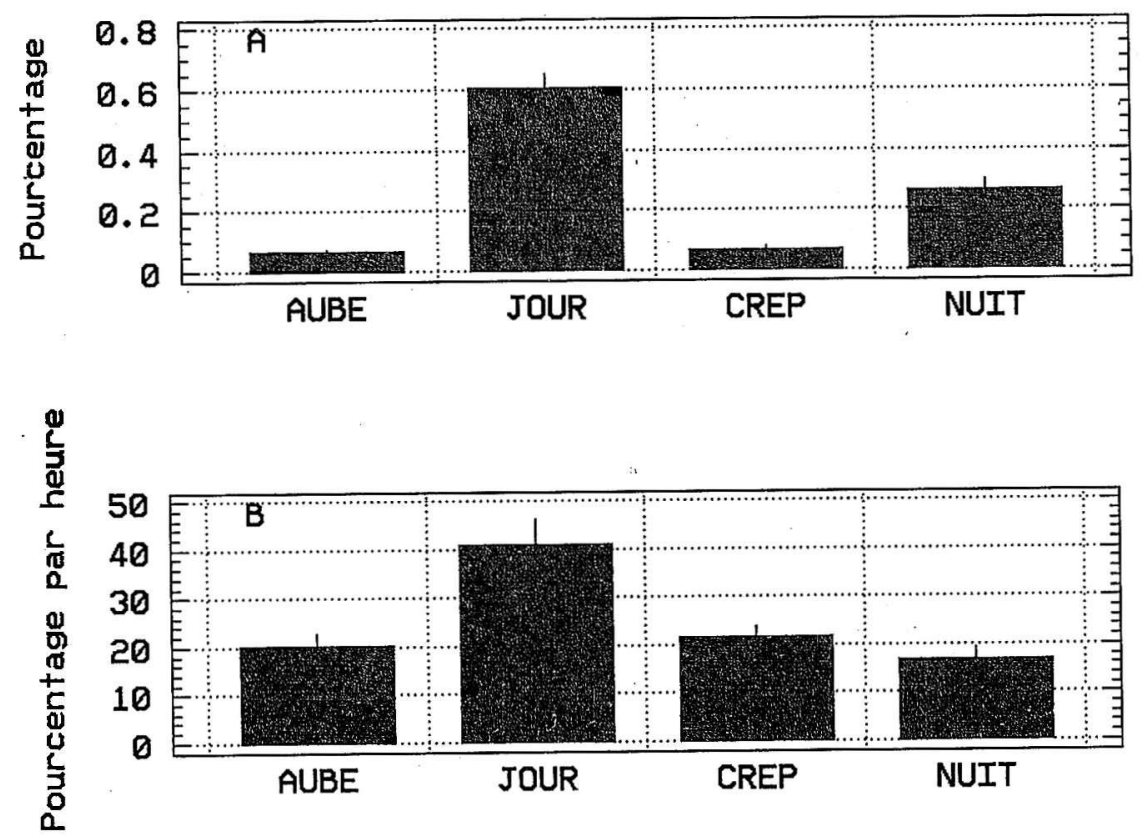

Figure 6A

Pourcentage de présence des saumons au pied de l'obstacle au cours de I: journée.

Figure 6B

Pourcentage de présence des saumons au pied de l'obstacle au cours de chaqu période du nycthémère rapportée aux durées de ces périodes.

\section{Figure 6A}

Percentage of presence of fish in the vicinity of the obstacle in relation to th time of the day.

Figure 6B

Percentage of presence of fish per hour in each diurnal period in the vicinity $\mathrm{C}$ the obstacle. 


\section{Heures d'arrivée et de départ du site}

II a été possible d'examiner la distribution des arrivées et des départs à l'échelle r. raire, respectivement 373 et 406 horaires d'arrivée et de départ du site ayant été $\epsilon$ registrés pour l'ensemble des 19 poissons étudiés (Figure 7). Seules les arrivées p. jcédées d'un départ à l'aval au moins égal à 6 heures et les départs supérieurs à $\epsilon$ ieures ont été pris en compte dans l'analyse. En effet, lors de la majorité des replis de c. urte durée, les saumons stationnaient généralement à proximité immédiate des zones c réception individualisées au pied de l'obstacle.

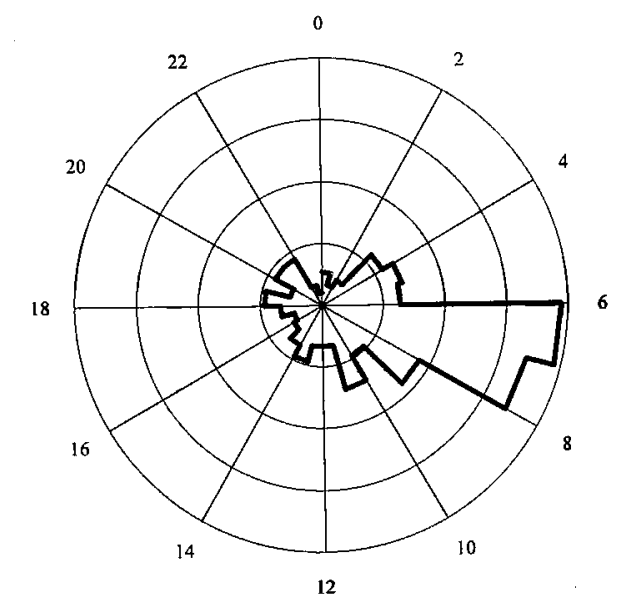

A

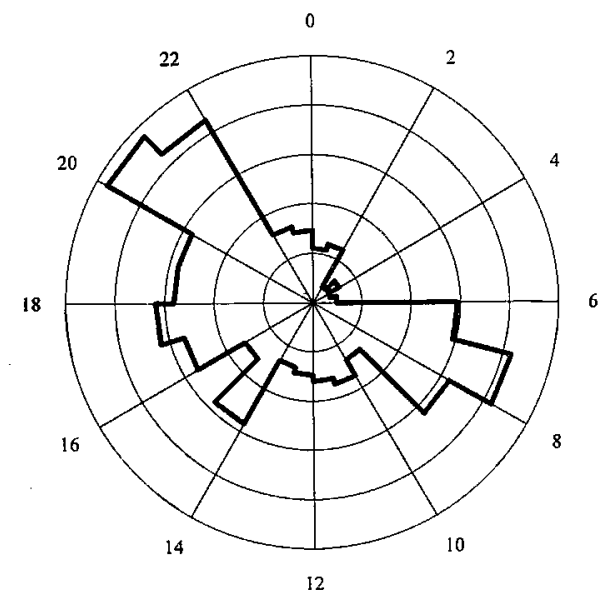

B

\section{Figure 7A}

Distribution des incursions au pied de l'obstacle au cours de la journée.

\section{Figure 7B}

Distribution des replis à l'aval au cours de la journée.

\section{Figure 7A}

Distribution of approaches in the vicinity of the plant in relation to the time of the day.

\section{Figure 7B}

Distribution of downstream departures in relation to the time of the day. 
Les poissons se présentent préférentiellement à l'aube $\left(\chi^{2}=146,7 ; p<0,000\right.$ : entre 6 et 8 heures, au pied de l'aménagement et quittent le site au crépusclie $\left(\chi^{2}=104,8 ; p<0,0001\right)$. Les départs sont cependant beaucoup plus étalés au cours de !a journée que les arrivées, une fraction non négligeable s'effectuant le matin, correspondant aux incursions de faibles durées sur le site.

\section{Influence de la température et du débit sur la présence au pied de l'obstacle}

Des analyses par régression multiple pas à pas indiquent d'une part que is nombres d'incursions par jour des poissons au niveau de l'aménagement dépendent de a température de l'eau $(p=0,0001)$ et d'autre part que les durées de présence au pied (e l'obstacle sont significativement liées aux débits du gave $(p=0,015)$.

II apparaît que les saumons se présentent plus fréquemment sur le site d'étuce lorsque les températures sont élevées (ANOVA à 1 facteur, avec le facteur températu e (a : $8-12^{\circ} \mathrm{C} ; b: 12-16^{\circ} \mathrm{C} ; c: 16-20^{\circ} \mathrm{C} ; d: 20-24^{\circ} \mathrm{C}$ ), $F_{3,856}=14,6 ; p<0,0001$ ), notamme $1 \mathrm{t}$ lorsqu'elles deviennent supérieures à $16^{\circ} \mathrm{C}$ (Figure $8 \mathrm{~A}$ ).

Les poissons stationnent plus longuement au pied de l'ouvrage pour des débiis faibles à moyens (ANOVA à 1 facteur, avec le facteur débit $\left(a:<50 \mathrm{~m}^{3} / \mathrm{s} ; b: 50-100 \mathrm{~m}^{3} / \mathrm{s}\right.$; $\left.\left.c:>100 \mathrm{~m}^{3} / \mathrm{s}\right), \mathrm{F}_{2,885}=6,2 ; \mathrm{p}<0,005\right)$, les durées de présence diminuant pour des débits supérieurs à $100 \mathrm{~m}^{3} / \mathrm{s}$, conditions hydrologiques qui correspondent généralement à des coups d'eau associés à des augmentations de la turbidité (Figure 8B).

Si l'on ne prend en considération que les jours où les saumons se sont présentés au pied de l'aménagement, une régression multiple pas à pas indique que seule la température de l'eau contrôle les durées de présence des poissons au pied de l'obstacle au cours de la journée ( $p<0,0001$ ). Ces derniers stationnent d'autant plus longuement au pied de l'obstacle que les températures sont faibles (ANOVA à 1 facteur, $F_{3,520}=16,8$; $p<0,0001$ ) (Figure $8 C$ ).

\section{Comportement des poissons au pied de l'obstacle}

Dans toutes les analyses ultérieures, seuls les jours où les poissons se sont présentés au pied de l'aménagement seront pris en compte.

Les températures et les débits turbinés ont été répartis respectivement en 4 et 2 catégories : $8-12^{\circ} \mathrm{C}, 12-16^{\circ} \mathrm{C}, 16-20^{\circ} \mathrm{C}$ et $20-24^{\circ} \mathrm{C}$ en ce qui concerne les températures et $0-50 \mathrm{~m}^{3} / \mathrm{s}$ et $50-90 \mathrm{~m}^{3} / \mathrm{s}$ pour les débits turbinés par l'usine hydroélectrique. L'usine pouvant turbiner jusqu'à $90 \mathrm{~m}^{3} / \mathrm{s}$, les déplacements des poissons au pied de l'obstacle ont été étudiés sans déversement au niveau du barrage.

\section{Présence et durées des incursions dans les différentes zones de réception}

Huit poissons ont stationné préférentiellement dans le canal de fuite de l'usine (zone de réception I), 5 au pied du barrage (zone de réception III), 3 des temps équivalents dans ces deux zones et 3 enfin dans la zone II située dans le prolongement de la jetée et qui présente la particularité d'être hydrauliquement abritée tout en étant à proximité du courant principal.

Les durées des incursions des poissons dans les zones II et III sont courtes, respectivement $89,2 \%$ (1 422 sur 1593 ) et 68,2\% (806 sur 1 182) étant inférieures à 1 heure. Ces durées sont plus importantes dans la zone I, 58,8\% (880 sur 1496 ) ne dépassant pas 1 heure mais $36 \%$ d'entre elles étant comprises entre 3 et 6 heures (Figure 9). Ces résultats mettent en évidence le fait que le canal de fuite paraît être la zone la plus attractive. 

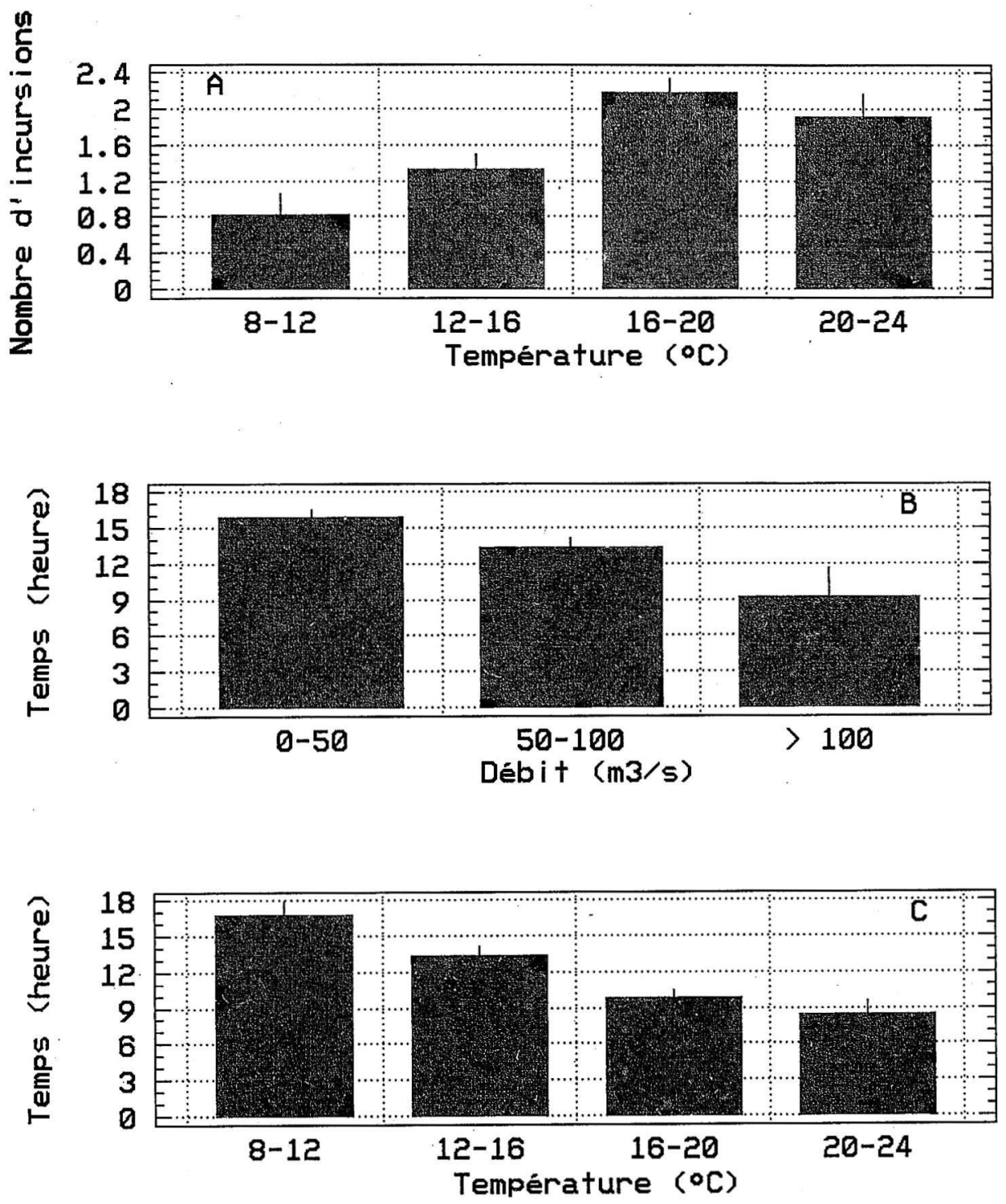

Figure 8A

Nombre moyen d'incursions par jour au pied de l'aménagement en fonction de la température de l'eau.

Figure 8B

Durées moyennes de présence par jour au pied de l'obstacle en fonction du débit.

Figure 8C

Durées moyennes de stationnement au pied de l'obstacle des poissons en fonction de la température.

Figure 8A

Mean daily number of approaches to the plant in relation to water temperature.

Figure 8B

Mean daily presence duration in the vicinity of the obstacle in relation to river flows.

Figure $8 \mathrm{C}$

Mean presence duration in the vicinity of the obstacle in relation to water temperature. 

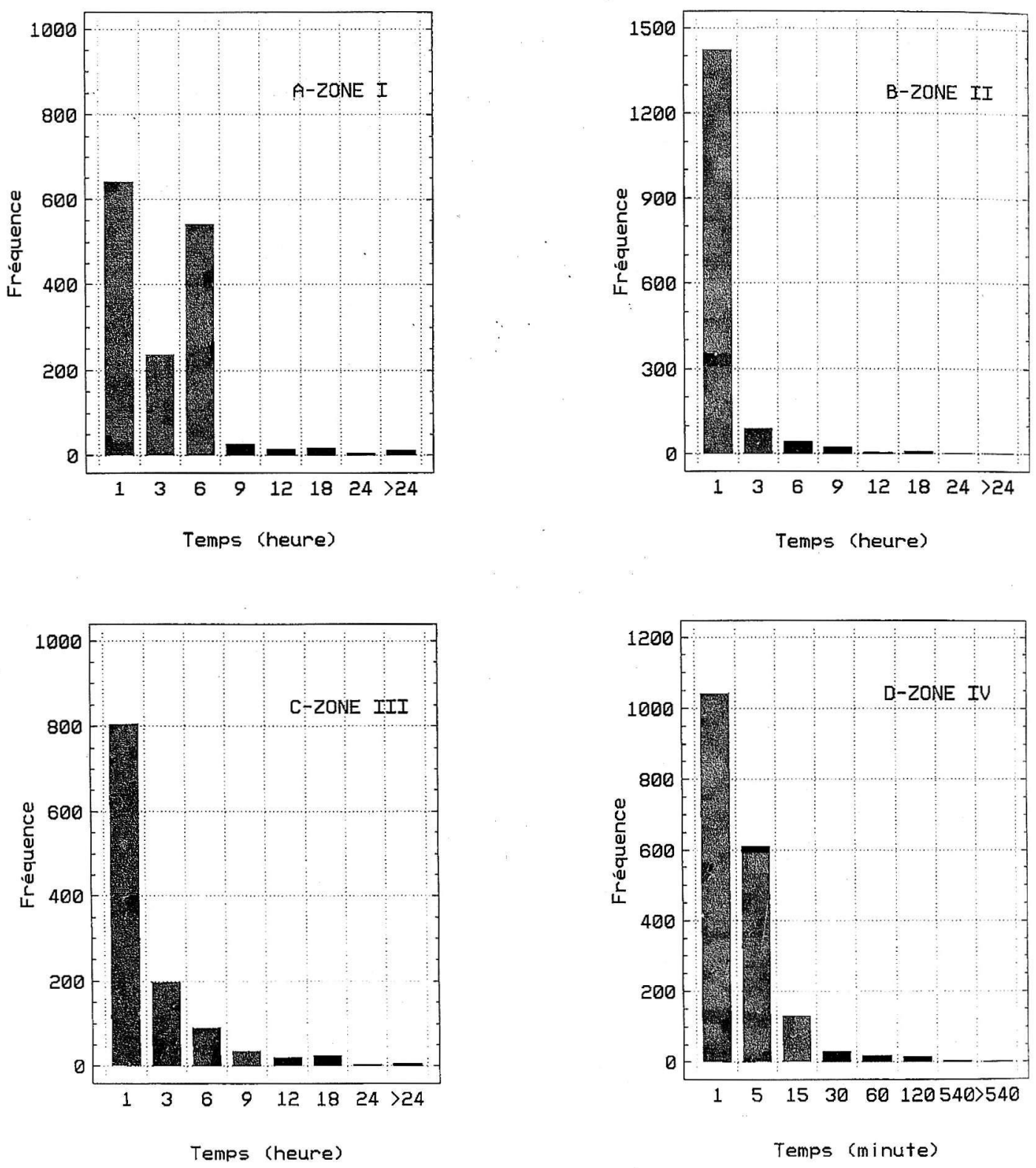

Figure 9A

Nombre et durée des incursions dans la zone I.

Figure 9B

Nombre et durée des incursions dans la zone II.

Figure 9C

Nombre et durée des incursions dans la zone III.

Figure 9D

Nombre et durée des incursions dans la zone IV.

Figure 9A

Number and duration of the visits to area $I$.

Figure 9B

Number and duration of the visits to area II.

Figure 9C

Number and duration of the visits to area III.

Figure 9D

Number and duration of the visits to area IV. 


\section{Influence de la température de l'eau et du fonctionnement de l'usine}

Les effets des facteurs débit turbiné et température de l'eau sur les pourcentages de prisence des saumons dans les différentes zones de réception individualisées au pied de l'a nénagement ont été étudiés à l'aide d'analyses de variance (ANOVA) à 2 facteurs (T. bleaux II et III).

Il apparaît clairement que la présence des poissons dans le canal de fuite est ne jement plus faible lorsque les températures dépassent $20^{\circ} \mathrm{C}$. Les poissons ont st ionné en moyenne entre 39 et $45,5 \%$ de leur temps de présence sur le site dans le ca ral pour des températures de l'eau inférieures à $20^{\circ} \mathrm{C}$ et $15,1 \%$ à peine lorsqu'elles de riennent supérieures. Le fonctionnement de l'usine hydroélectrique n'exerce aucune ini lence, le canal restant toujours, quels que soient les débits, une zone attractive.

Les pourcentages de présence des saumons à l'aval immédiat de la jetée (zone II), zo ie hydrauliquement protégée mais à proximité immédiate du courant principal, sont plus im ortants lorsque les débits turbinés sont supérieurs à $50 \mathrm{~m}^{3} / \mathrm{s}$, les poissons y stationnant de: is ces conditions plus de $25 \%$ contre $19 \%$ pour des débits inférieurs.

Les poissons stationnent proportionnellement plus de temps au pied du barrage (zcne III) lorsque les débits turbinés sont inférieurs à $50 \mathrm{~m} 3 / \mathrm{s}$ : les poissons y ont passé en moyenne près de $45 \%$ de leur temps de présence au pied de l'obstacle contre à peine $37 \%$ pour des débits supérieurs. Les effets de la température sur les pourcentages de presence dans la zone III s'expliquent par le fait que les poissons stationnent moins au pied de l'aménagement et dans le canal de fuite pour des températures élevées et non par le fait qu'ils stationnent plus au pied du barrage.

Le fonctionnement de l'usine exerce un effet sur la présence des poissons au pied du dispositif de franchissement, les poissons séjournant en moyenne $2,4 \%$ de leur temps au pied de la passe à poissons quand les débits sont inférieurs à $50 \mathrm{~m}^{3} / \mathrm{s}$ et $5,1 \%$ pour des débits plus élevés. L'influence de la température, mise en évidence par l'analyse statistique, ne paraît pas toutefois très claire.

\section{Comportement au niveau de l'entrée du dispositif de franchissement}

Au niveau de l'entrée du dispositif de franchissement (zone IV), $56,2 \%$ et $89,2 \%$ (1 041 sur 1851 et 1652 sur 1851 ) des incursions sont respectivement inférieures à 1 et 5 minutes (Figure 9D). Ce résultat est dû en partie à la faible superficie de cette zone $\left(6 \mathrm{~m}^{2}\right.$ environ), les poissons pouvant quitter la zone de réception pour de faibles déplacements, mais aussi, très certainement, à sa faible attractivité.

Tous les poissons se sont présentés de nombreuses fois au niveau de l'entrée du dispositif, en moyenne 91 fois (médiane : 40 fois). De plus, les poissons ont été reçus généralement au niveau de l'entrée du dispositif de franchissement peu de temps après leur arrivée initiale sur le site, 18 des 19 saumons radiomarqués ayant mis en moyenne 12,5 heures (médiane : 6,4 heures). Ce délai représenterait la durée minimale de blocage des poissons induite par l'aménagement dans l'hypothèse où l'efficacité hydraulique de la passe à poissons serait optimale. Le dernier poisson n'est resté que quelques heures au pied de l'obstacle lors de son arrivée initiale puis s'est replié à l'aval, dans un profond, pendant près de 52 jours. Lors de son retour sur le site, fin octobre, il n'a mis que 30 minutes pour se présenter au pied de l'échelle.

Seize des 1851 réceptions au niveau de l'entrée de la passe à poissons, soit $0,86 \%$, ont été suivies d'une incursion à l'intérieur du dispositif de franchissement. Le nombre médian d'incursions au pied de l'échelle nécessaires pour le franchissement de l'obstacle est de $43 ; 4$ saumons ont même effectué plus de 100 incursions au pied du dispositif de franchissement avant de passer à l'amont. Tous les poissons, qu'ils aient ou non franchi l'aménagement, ont stationné dans la zone IV, un saumon s'y présentant même plus de 200 fois sans réussir à passer à l'amont. 


\section{Tableau II}

Pourcentage de présence des saumons dans la zone I (canal de fuite) et dans zone Il (aval jetée) en fonction de la température de l'eau et du débit turbiné.

\section{Table II}

Percentage of presence of fish in area I (tailrace) and area II (downstream the pis in relation to water temperature and turbine discharge.

\begin{tabular}{ccccc}
\hline $\begin{array}{c}\text { Pourcentage de présence } \\
\text { dans la zone I (canal de fuite) }\end{array}$ & DEBIT $<50 \mathrm{~m}^{3} / \mathrm{s}$ & $\mathrm{N}$ & DEBIT $>50 \mathrm{~m}^{3} / \mathrm{s}$ & \\
\hline $8^{\circ} \mathrm{C}<$ TEMPERATURE $\leq 12^{\circ} \mathrm{C}$ & $0,67 \pm 0,09$ & 25 & $0,66 \pm 0,11$ & 1 \\
$12^{\circ} \mathrm{C}<$ TEMPERATURE $\leq 16^{\circ} \mathrm{C}$ & $0,70 \pm 0,04$ & 111 & $0,65 \pm 0,08$ & 3 \\
$16^{\circ} \mathrm{C}<$ TEMPERATURE $\leq 20^{\circ} \mathrm{C}$ & $0,62 \pm 0,05$ & 99 & $0,64 \pm 0,05$ & 7 \\
$20^{\circ} \mathrm{C}<$ TEMPERATURE $\leq 24^{\circ} \mathrm{C}$ & $0,17 \pm 0,07$ & 44 & $0,37 \pm 0,10$ & 2 \\
\hline ANOVA à 2 facteurs : Température $: p<0,0001 ;$ Débit $: p=0,56 ;$ Interaction $: p=0,45$
\end{tabular}

\begin{tabular}{ccccc}
\hline $\begin{array}{c}\text { Pourcentage de présence } \\
\text { dans la zone II (aval jetée) }\end{array}$ & DEBIT $<50 \mathrm{~m}^{3} / \mathrm{s}$ & $\mathrm{N}$ & DEBIT $>50 \mathrm{~m}^{3} / \mathrm{s}$ & $\mathrm{N}$ \\
\hline $8^{\circ} \mathrm{C}<$ TEMPERATURE $\leq 12^{\circ} \mathrm{C}$ & $0,22 \pm 0,08$ & 25 & $0,6 \pm 0,10$ & 18 \\
$12^{\circ} \mathrm{C}<$ TEMPERATURE $\leq 16^{\circ} \mathrm{C}$ & $0,38 \pm 0,04$ & 111 & $0,44 \pm 0,07$ & 33 \\
$16^{\circ} \mathrm{C}<$ TEMPERATURE $\leq 20^{\circ} \mathrm{C}$ & $0,37 \pm 0,04$ & 99 & $0,45 \pm 0,05$ & 75 \\
$20^{\circ} \mathrm{C}<$ TEMPERATURE $\leq 24^{\circ} \mathrm{C}$ & $0,31 \pm 0,06$ & 44 & $0,48 \pm 0,09$ & 20 \\
\hline
\end{tabular}

ANOVA à 2 facteurs : Température $: p=0,99 ;$ Débit : $p<0,001 ;$ Interaction : $p=0,22$

(Les résultats correspondent aux pourcentages de présence transformés par la fonction Arcsinv

\section{Tableau III}

Pourcentage de présence des saumons dans la zone III (barrage) et dans li zone IV (entrée passe) en fonction de la température de l'eau et du débit turbiné.

\section{Table III}

Percentage of presence of fish in area III (dam) and area IV (fishpass entrance) iI relation to water temperature and turbine discharge.

\begin{tabular}{ccccr}
\hline $\begin{array}{c}\text { Pourcentage de présence } \\
\text { dans la zone III (barrage) }\end{array}$ & DEBIT $<50 \mathrm{~m}^{3} / \mathrm{s}$ & $\mathrm{N}$ & DEBIT $>50 \mathrm{~m}^{3} / \mathrm{s}$ & $\mathrm{N}$ \\
\hline $8^{\circ} \mathrm{C}<$ TEMPERATURE $\leq 12^{\circ} \mathrm{C}$ & $0,71 \pm 0,09$ & 25 & $0,42 \pm 0,11$ & 18 \\
$12^{\circ} \mathrm{C}<$ TEMPERATURE $\leq 16^{\circ} \mathrm{C}$ & $0,58 \pm 0,04$ & 111 & $0,57 \pm 0,08$ & 33 \\
$16^{\circ} \mathrm{C}<$ TEMPERATURE $\leq 20^{\circ} \mathrm{C}$ & $0,66 \pm 0,04$ & 99 & $0,58 \pm 0,05$ & 75 \\
$20^{\circ} \mathrm{C}<$ TEMPERATURE $\leq 24^{\circ} \mathrm{C}$ & $1,13 \pm 0,07$ & 44 & $0,76 \pm 0,10$ & 20 \\
\hline ANOMARER
\end{tabular}

ANOVA à 2 facteurs : Température : $p<0,0001 ;$ Débit : $p<0,005 ;$ Interaction : $p=0,07$

\begin{tabular}{ccccr}
\hline $\begin{array}{c}\text { Pourcentage de présence } \\
\text { dans la zone IV (entrée passe) }\end{array}$ & DEBIT $<50 \mathrm{~m}^{3} / \mathrm{s}$ & $\mathrm{N}$ & DEBIT $>50 \mathrm{~m}^{3} / \mathrm{s}$ & $\mathrm{N}$ \\
\hline $8^{\circ} \mathrm{C}<$ TEMPERATURE $\leq 12^{\circ} \mathrm{C}$ & $0,10 \pm 0,027$ & 25 & $0,067 \pm 0,03$ & 18 \\
$12^{\circ} \mathrm{C}<$ TEMPERATURE $\leq 16^{\circ} \mathrm{C}$ & $0,05 \pm 0,01$ & 111 & $0,076 \pm 0,024$ & 33 \\
$16^{\circ} \mathrm{C}<$ TEMPERATURE $\leq 20^{\circ} \mathrm{C}$ & $0,066 \pm 0,014$ & 99 & $0,15 \pm 0,12$ & 75 \\
$20^{\circ} \mathrm{C}<$ TEMPERATURE $\leq 24^{\circ} \mathrm{C}$ & $0,076 \pm 0,02$ & 44 & $0,19 \pm 0,03$ & 20 \\
\hline ANOMA
\end{tabular}

ANOVA à 2 facteurs : Température $: p=0,01$; Débit : $p<0,005 ;$ Interaction : $p=0,013$ (Les résultats correspondent aux pourcentages de présence transformés par la fonction Arcsim 


\section{Activité au pied de l'obstacle}

Le nombre d'incursions dans les zones I et III rapporté à l'heure de présence des poissons sur le site a semblé être un bon indicateur de l'activité des saumons au pied de l'ostacle.

Les poissons se sont montrés nettement moins actifs au pied de l'obstacle de nuit q ie lors des autres périodes du nycthémère : nuit-aube $(W, Z=2,70 ; p<0,01)$, nuit-jour $(\mathrm{l}, Z=2,71 ; p<0,01)$ et nuit-crépuscule $(W, Z=2,67 ; p<0,01)$, aucune différence $n$ xyant été mise en évidence entre les trois autres périodes (Figure 10A).

Les saumons ont été plus actifs au pied de l'aménagement lorsque les températures ot dépassé $16^{\circ} \mathrm{C}$ (ANOVA, $F_{3,428}=5,78 ; p=0,0007$ ), effectuant en moyenne moins de $0 ?$ incursion par heure pour des températures inférieures à $16{ }^{\circ} \mathrm{C}$ contre plus de 1 incursion pour des températures supérieures (Figure 10B).
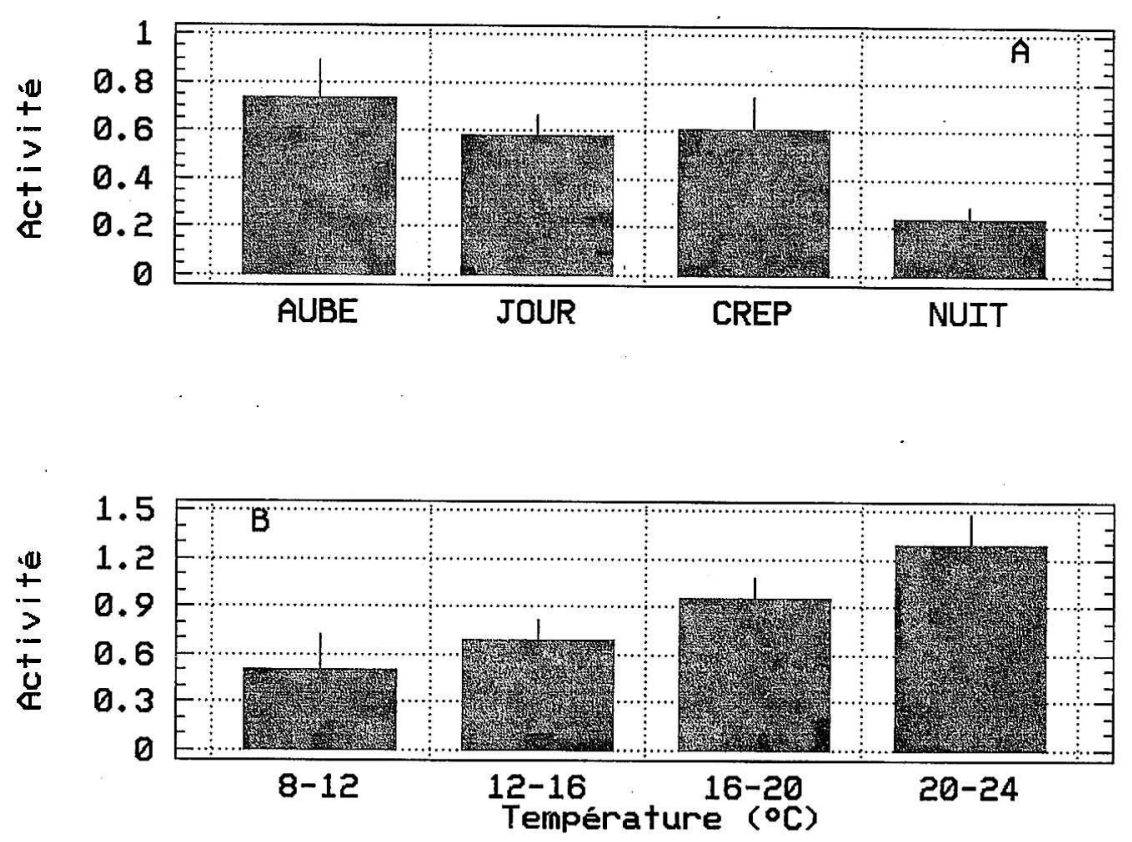

Figure 10A

Activité journalière des poissons au pied de l'obstacle.

Figure 10B

Activité des poissons au pied de l'obstacle en fonction de la température de l'eau.

\section{Figure 10A}

Daily activity of fish in the vicinity of the obstacle.

\section{Figure 10B}

Activity of fish in the vicinity of the obstacle in relation to water temperature. 


\section{Tableau IV}

Nombre moyen d'incursions par jour au niveau de l'aménagement, activis moyenne journalière au pied de l'obstacle et présence moyenne journalière niveau de l'entrée de la passe à poissons pour les poissons qui sont parvenus i franchir l'obstacle et ceux qui n'ont pu passer à l'amont.

\section{Table IV}

Mean daily number of approaches to the plant, mean daily activity in the vicin ? of the obstacle and mean daily presence at the fish pass entrance for fish whic passed over the plant and those which did not.

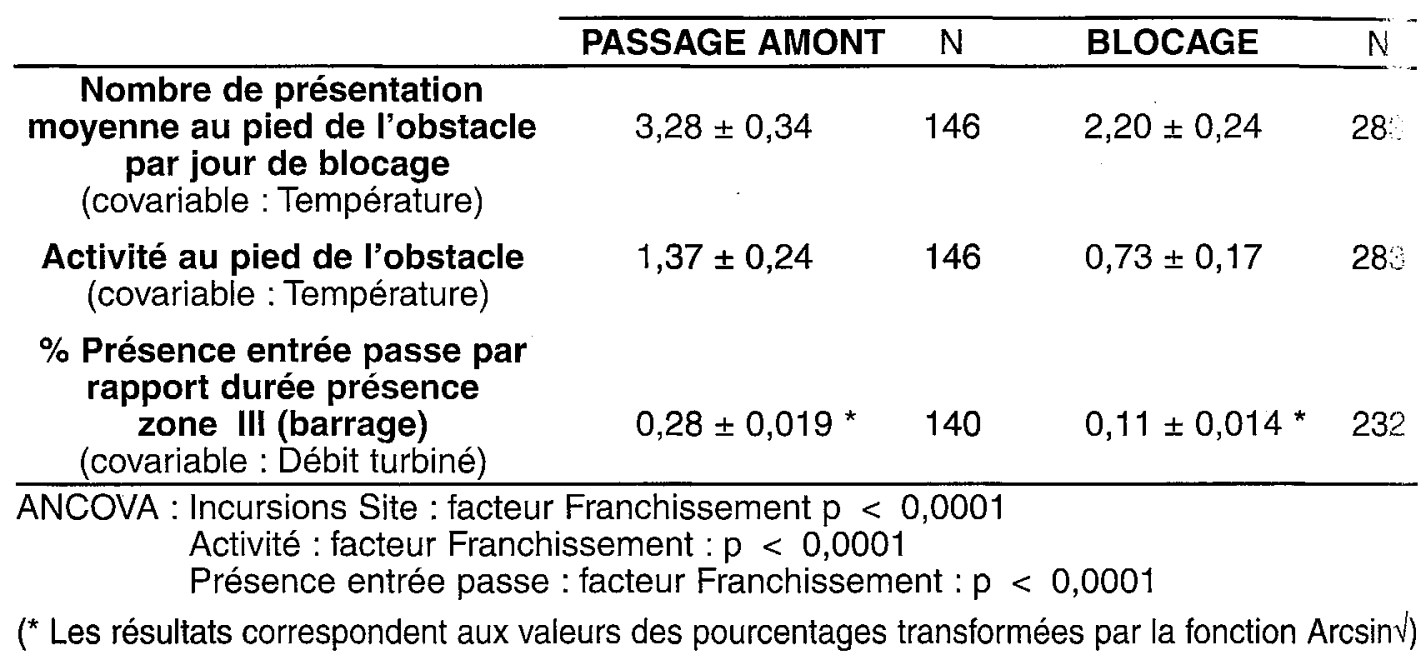

\section{Franchissement de l'obstacle}

Des analyses de covariance (ANCOVA) ont permis de mettre en évidence des différences de comportement entre les poissons qui ont franchi l'aménagement et ceux qui n'y sont pas parvenus (Tableau IV), notamment en ce qui concerne le nombre d'incursions des poissons au pied de l'obstacle $(p<0,0001)$, leur activité au pied de l'obstacle, mesurée par le nombre de changements de zone par heure de présence $(p<0,0001)$ et le pourcentage de temps passé au niveau de l'entrée de la passe à poissons par rapport au temps de présence dans la zone barrage $(p<0,0001)$.

II apparaît que les saumons qui ont franchi l'aménagement se sont présentés en moyenne 3,3 fois par jour au pied de l'obstacle contre 2,2 fois pour ceux qui n'ont pu passer à l'amont.

De même, ils ont été près de 2 fois plus actifs au pied de l'obstacle réalisant en moyenne 1,37 changement de zone par heure de présence sur le site contre à peine 0,73 pour les saumons qui sont restés à l'aval.

Enfin, les poissons qui ont réussi à franchir l'obstacle ont stationiné proportionnellement plus de temps $(12,8 \%)$ au niveau de l'entrée du dispositif de franchissement que les autres saumons $(4,3 \%)$ lorsqu'ils étaient présents dans la zo:e barrage (zone III). 


\section{Comportement dans la passe à poissons}

Sur les 16 incursions au pied de la passe à poissons qui ont été suivies d'une incursion dans le dispositif, 13 se sont déroulées en pleine journée, 2 au crépuscule et 1 de nuit. Cinq de ces incursions n'ont pas été suivies d'un franchissement, les poissons ridévalant la passe à poissons après des durées variables de présence comprises entre (i) minutes et plus de 7 heures, 4 d'entre elles ne dépassant pas cependant 1 heure. L'une ìs 2 incursions à l'intérieur de la passe à poissons effectuée au crépuscule ainsi que c.lle réalisée de nuit n'ont pas été suivies du franchissement du dispositif. Lors de la conde incursion réalisée au crépuscule, le saumon a passé la nuit entière dans le 2ème, c.me ou 4ème bassin de repos et a été reçu le lendemain, en milieu de matinée, dans le cərnier bassin de repos, avant de franchir l'aménagement en fin de matinée. La f: anchissabilité du dispositif ne paraît pas optimale, 5 incursions à l'intérieur du dispositif $r$ 'ayant pas été suivies de son franchissement.

Les durées de présence dans la passe à poissons lors de 8 des 11 incursions à l'atérieur de la passe à poissons suivies du franchissement de l'aménagement ont pu être ćterminées. Sans prendre en compte le poisson qui est resté 17 heures dans la passe à pissons de nuit, 4 individus sont restés moins de 1 heure 30 à l'intérieur du dispositif, 1 y a séjourné 2 heures et 2 enfin ont franchi la passe à poissons en 3 heures.

Les saumons stationnent généralement peu de temps dans le premier bassin de repos : deux seulement sur les 10 durées enregistrées ont été supérieures à 4 minutes ( $f$ et 13 minutes).

Les durées dans les deuxième et troisième bassins sont homogènes et plus importantes qu'au niveau du premier bassin, 6 des 8 temps de stationnement étant compris entre 10 et 20 minutes pour une moyenne de 13,3 minutes.

Les durées de présence dans le quatrième bassin sont nettement plus élevées que dans les autres bassins aval : un saumon sur les 4 poissons reçus n'a été reçu que 9 minutes, les 3 autres ayant stationné plus de 30 minutes (moyenne : 44,7 minutes). La chute relativement importante (entre 45 et $55 \mathrm{~cm}$ ) entre ce bassin de repos et le bassin à parois déversantes amont peut expliquer les durées importantes de stationnement des saumons. L'observation visuelle du saumon radiomarqué qui est resté le moins longtemps dans ce bassin a mis en évidence un des comportements caractéristiques d'un poisson au pied d'une chute. Ce saumon a sorti la tête au moins 2 fois hors de l'eau au pied de la chute avant de passer à l'amont, dans la lame déversante.

Les durées de présence dans le cinquième bassin de repos sont peu homogènes et varient de 12 minutes à plus d'une heure, la moyenne étant de 32,4 minutes. Trois des 6 durées enregistrées sont comprises entre 12 et 25 minutes.

\section{DISCUSSION - CONCLUSION}

Trente-deux des 43 saumons marqués et lâchés à Puyoo sont parvenus jusqu'à l'aménagement de Baigts, situé 8 kilomètres à l'amont, les délais variant de 8 heures à près de 49 jours. Cette hétérogénéité dans les résultats est comparable à celle obtenue par d'autres auteurs, en particulier SMITH, JOHNSTONE et SHEARER (1996), qui ont mis en évidence des délais allant de 5 à 57 jours, les poissons étant pourtant lâchés à la même date et le point de lâcher n'étant distant de l'obstacle que de 1 kilomètre. 
Sur ces 32 poissons, 2 saumons ont régurgité leur émetteur $(4,6 \%)$. Ce taux cie régurgitation est plus faible que ceux mis en évidence par SMITH, CAMPBELL at MACLAINE (1998) qui étaient compris entre $12,5 \%$ et $16,7 \%$.

Quatre poissons, bloqués par l'aménagement de Baigts, ont dévalé et ont ensuil été retrouvés sur d'autres rivières, deux sur le gave d'Oloron et deux sur un de ses affluents, le Saison. Un saumon, marqué fin juillet à Puyoo, a dévalé peu de temps après le lâcher puis est resté plus de 15 jours au niveau de la confluence du gave de Pau et $c$ u gave d'Oloron avant de retourner en mer et d'être piégé fin novembre sur la Nivelle, ć. niveau d'une station de contrôle. D'autres études ont révélé de tels comportemenis (LAUGHTON, 1991 ; STONEHEWER, WALTERS et MEE, 1993), certains poissor: pouvant remonter un cours d'eau sur plus de 20 kilomètres avant de dévaler (MILNEF: 1990).

Seulement $34 \%$ des poissons qui se sont présentés au pied de l'aménagement on: réussi à passer à l'amont, les durées de blocage pouvant atteindre près de 80 jours. Le: poissons qui n'ont pas franchi l'obstacle sont restés bloqués jusqu'à 137 jours à l'aval de l'ouvrage. L'impact de cet obstacle sur la migration des saumons, à la fois en terme ds pourcentage et de retard à la migration, est incompatible avec tout plan de restauration di saumon sur le gave de Pau.

La majorité des poissons a stationné bien à l'aval de l'obstacle au cours du blocage dans un profond s'étendant de $500 \mathrm{~m}$ à 1,2 kilomètre à l'aval de l'obstacle. $\mathrm{C} \epsilon$ comportement est vraisemblablement lié à la topographie du site d'étude, aucune zone dt stationnement propice ne se trouvant à proximité de l'aménagement.

Les temps de présence en continu des poissons sur le site et les départs à l'ava étaient généralement de courtes durées, inférieures à 1 heure, les saumons se repliant at maximum à 200-300 $\mathrm{m}$ à l'aval de l'ouvrage et stationnant préférentiellement en rive gauche, en limite du courant principal. Pour des replis de durées plus importantes supérieures à 6 heures, les poissons étaient très souvent localisés dans le profonc mentionné plus haut.

II est généralement admis que la migration du saumon atlantique dans les parties non obstruées des cours d'eau se déroule principalement de nuit (BANKS, 1969 ; SMITH 1985 ; HAWKINS et SMITH, 1986 ; WEBB, 1989 ; LAUGHTON, 1991 ; MILNER, 1990 JONSSON, 1991), des déplacements vers l'amont pouvant toutefois avoir lieu en pleint journée en période de crue (HELLAWELL, LEATHAM et WILLIAMS, 1974 ; LAUGHTON 1989). Par contre, au niveau des obstacles, très peu de données concernant lf comportement des poissons ont été recueillies. Les seules données accessibles sont it plus souvent les chronologies des passages dans les dispositifs de franchissement passages qui semblent être majoritairement diurnes dans les passes à bassint (DARTIGUELONGUE et LANGON, 1998) et variables au niveau de seuils de comptagt (DUNKLEY et SHEARER, 1982 ; SMITH, JOHNSTONE et DUNKLEY, 1996 ; SMITH e SMITH, 1997). Ces résultats quelque peu contradictoires sont certainement plus le refle de la distribution temporelle, et en particulier nycthémérale, des conditions permettant au: poissons de franchir l'obstacle que de la variation temporelle de leur motivation à migre (CRISP, 1996).

Lors des trois années d'expérimentation, la grande majorité des poissons a étı présente en pleine journée au pied de l'aménagement, aucun poisson n'ayant stationn préférentiellement de nuit au niveau de l'obstacle. De plus, l'activité des poissons a étı nettement supérieure de jour au pied de l'ouvrage. 
Les poissons ont semblé présenter une certaine rythmicité comportementale au cours du nycthémère. Les arrivées au pied de l'obstacle, après des départs supérieurs à E. heures, se sont effectuées majoritairement à l'aube, les poissons se présentant très rirement de nuit au niveau de l'aménagement. Ce résultat est comparable à celui obtenu far SMITH, JOHNSTONE et SHEARER (1996) mais semble différent de celui de STONEHEWER, KIRKPATRICK et MEE (1995) qui n'ont pas mis en évidence de r. éférence au cours du nycthémère. Les départs ont été beaucoup plus étalés que les c rivées, un nombre important d'entre eux survenant en fin de journée, au crépuscule, une faction non négligeable s'effectuant toutefois le matin, correspondant aux incursions de $f$ bles durées sur le site.

II semblerait ainsi y avoir chez le saumon atlantique, comme l'avance BANKS (969), un conflit entre le besoin de lumière pour franchir les obstacles et une préférence four la nuit sur les parties de cours d'eau sans obstacle.

La présence des poissons au niveau de l'aménagement de Baigts est apparue liée à li: température moyenne journalière de l'eau, les saumons se présentant plus fuquemment au pied de l'obstacle lorsque les températures étaient supérieures à $16^{\circ} \mathrm{C}$. Les saumons étaient également plus actifs au pied de l'aménagement. Il est généralement admis que l'activité augmente avec les températures de l'eau (JONSSON, 1991) jusqu'à wine certaine valeur variant, selon les auteurs, entre $22^{\circ} \mathrm{C}$ et $24-25^{\circ} \mathrm{C}$ (ELSON, 1969 ; A.ABASTER, 1990). Les poissons stationnaient toutefois moins longtemps au pied de l'ubstacle et en particulier dans le canal de fuite, révélant peut être un preferendum thermique de valeur inférieure.

Les saumons ont stationné moins de temps au pied de l'obstacle lorsque les débits étaient supérieurs à $100 \mathrm{~m} 3 / \mathrm{s}$. Ces conditions hydrologiques correspondent à des coups d'eau généralement associés à une augmentation de la turbidité.

Le canal de fuite, par où transite le débit turbiné par l'usine, est resté une zone toujours attractive. Les poissons ont stationné toutefois nettement moins longtemps au pied de l'usine lorsque les températures de l'eau étaient élevées, supérieures à $20^{\circ} \mathrm{C}$.

La zone située à l'aval de la jetée, zone hydrauliquement protégée mais située à proximité du canal de fuite, a été plus fréquentée lorsque les débits turbinés étaient supérieurs à $50 \mathrm{~m}^{3} / \mathrm{s}$. Un comportement similaire de salmonidés au pied d'un obstacle a déjà été mis en évidence par RAINEY (1991).

Les durées de présence des saumons au pied du barrage ont été proportionnellement plus importantes lorsque les débits turbinés étaient inférieurs à $50 \mathrm{~m}^{3} / \mathrm{s}$.

Les poissons ont stationné plus longuement au niveau de l'entrée du dispositif de franchissement pour des débits turbinés supérieurs à $50 \mathrm{~m}^{3 / \mathrm{s}}$. La présence des poissons au pied de la passe à poissons est très certainement la conséquence d'un comportement exploratoire prononcé, les saumons devant en effet traverser la vaste zone de faible profondeur située à l'aval des vannes du barrage, généralement peu attractive car alimentée (hors période de déversement) uniquement par le débít de la passe à poissons et les fuites au niveau des vannes du barrage. Ces déplacements pourraient ainsi être facilités par des débits plus importants, généralement liés à des profondeurs d'eau plus importantes.

Les saumons qui ont franchi l'aménagement ont présenté un comportement différent de celui des poissons qui ne sont pas parvenus à passer à l'amont. Ils ont été plus actifs au niveau de l'aménagement, se présentant plus souvent au niveau de l'obstacle et se 
déplaçant davantage au pied de l'aménagement. De plus, ils ont été beaucoup pi:is présents au niveau de l'entrée du dispositif de franchissement révélant un comporteme:tt exploratoire plus marqué.

Tous les saumons se sont présentés plusieurs fois au pied de la passe à poissor s. De plus, les délais entre les arrivées initiales des poissons sur le site et leur venue u niveau de l'échelle étaient peu élevés (en moyenne 12,5 heures) pour la grande majorié des saumons.

La faible efficacité du dispositif de franchissement ne peut donc être uniquement attribuée à sa position, sur la berge opposée au canal de fuite de l'usine.

\section{REMERCIEMENTS}

Ces études ont bénéficié de l'aide technique de J.M. Bach, O. Croze, E. Galiay at F. Giroux ainsi que des équipes de piégeage de MIGRADOUR.

Nous remercions la Fédération Départementale des Associations Agréées de Pêche et de Protection des Milieux Aquatiques des Pyrénées Atlantiques, le Conseil Supérieur cle la Pêche, en particulier la Brigade Mobile d'Intervention de la Délégation Régionale Midi-Pyrénées, et Électricité De France, notamment le sous-groupe d'Oloron et le groupement de Baigts de Béarn.

\section{BIBLIOGRAPHIE}

ALABASTER J.S., 1990. The temperature requirements of adult Atlantic salmon, Salmc salar L., during their upstream migration in the River Dee. J. Fish Biol., 37, 659-661.

BANKS J.W., 1969. A review of the literature on the upstream migration of adull salmonids. J. Fish. Biol., 1, 85-136.

BARAS E., LAGARDERE J.P., 1995. Fish telemetry in aquaculture : review anc perspectives. Aquaculture International, 3, 77-102.

CHANSEAU M., CROZE O., LARINIER M., 1999. Impact des aménagements sur la migration anadrome du saumon atlantique (Salmo salar L.) sur le gave de Pal (France). Bull. Fr. Pêche Piscic., 353/354, 211-238.

CRISP D.T., 1996. Environmental requirements of common riverine european salmonic fish species in fresh water with particular reference to physical and chemica aspects. Hydrobiologia, 323, 201-221.

DARTIGUELONGUE J., LANGON M., 1998. Contrôle du fonctionnement de l'ascenseur $\grave{c}$ poissons de Tuilières en 1997. Suivi de l'activité ichtyologique. Rapport S.C.E.A pour MI.GA.DO., 33 p.

DUNKLEY D.A., SHEARER D.W., 1982. An assessment of the performance of a resistivity fish counter. J. Fish Biol., 20, 717-737.

ELSON P.F., 1969. High temperature and river ascent by Atlantic salmon. Anadromous and Catadromous Fish Committee, ICES. Dublin, Eire, 1969. C.M 1969/M : 12, 5 p.

HAWKINS A.D., SMITH G.W., 1986. Radio-tracking observations on Atlantic salmor ascending the Aberdeenshire Dee. Scottish Fisheries Research Report, 36, 24 p.

HELLAWELL J.M., LEATHAM H., WILLIAMS G.I., 1974. The upstream migrator behaviour of salmonids in the River Frome, Dorset. J. Fish Biol., 6, 729-744. 
JENSEN A.J., HEGGBERGET T.G., JOHNSEN B.O., 1986. Upstream migration of adult Atlantic salmon, salmo salar L., in the River Vefsna, northern Norway. J. Fish Biol., 29, 459-465.

JENSEN A.J., JOHNSEN B.O., HANSEN L.P., 1989. Effect of river flow and water temperature on the upstream migration of adult Atlantic salmon, Salmo salar L., in the River Vefsna, northern Norway. In : Proceedings of the second international symposium on salmonid migration and distribution, BRANNON E. and JONSSON B. (Eds.), Trondheim, Norway, 140-146.

J.NSEN A.J., HVIDSTEN N.A., JOHNSEN B.O., 1998. Effects of temperature and flow on the upstream migration of adult Atlantic salmon in two Norwegian Rivers. In : Fish migration and fish bypasses, JUNGWIRTH M., SCHMUTZ S. and WEISS S. (Eds.), Fishing News Books, Blackwell Science Ltd, 45-54.

J)NSSON N., 1991. Influence of water flow, water temperature and light on fish migration in rivers. Nordic J. Freshw. Res., 66, 20-35.

K. NARD B.E., 1991. Anadromous fish behaviour important for fish passage. Proceedings of the workshop on fish passage at hydroelectric developments, WILLIAMS U.P., SCRUTON D.A., GOOSNEY R.F., BOURGEOIS C.E., ORR D.C. and RUGGLES C.P. (Eds.). Canadian Technical Report of Fisheries and Aquatic Sciences No. 1905, 95-105.

LEUGHTON R, 1989. The movements of adult salmon within the River Spey. Scottish Fisheries Research Report, 41, $19 \mathrm{p}$.

LAUGHTON R., 1991. The movements of adult Atlantic salmon (Salmo salar L.) in the River Spey as determined by radiotelemetry during 1988 and 1989. Scottish Fisheries Research Report, 50, $34 \mathrm{p}$.

MiLNER N.J. (ed.), 1990. Fish movements in relation to fresh water flow and quality. Proceedings of Atlantic Salmon Trust/Wessex Water Workshop, Pitlochry, Atlantic Salmon Trust, $51 \mathrm{p}$.

NORTHCOTE T.G., 1998. Migratory behaviour of fish and its significance to movement through riverine fish passage facilities. In : Fish migration and fish bypasses, JUNGWIRTH.M., SCHMUTZ S. and WEISS S. (Eds.), Fishing News Books, Blackwell Science Ltd, 3-18.

POTTER E.C.E., 1988. Movements of Atlantic salmon, Salmo salar L., in an estuary in south west England. J. Fish Biol., 33 sup. A, 153-159.

RAINEY W.S., 1991. Recent adult fish passage projects on tributaries of the Columbia River. Am. Fish. Soc. Symp., 10, 278-288.

SMITH R.J.F., 1985. The control of fish migration. Springer-Verlag, Berlin, Heidelberg, New York, Tokyo, $243 \mathrm{p}$.

SMITH I.P., SMITH G.W., 1997. Tidal and diel timing of river entry by adult Atlantic salmon returning to the Aberdeenshire Dee, Scotland. J. Fish Biol., 50, 463-474.

SMITH I.P., JOHNSTONE A.D.F., DUNKLEY D.A., 1996. Evaluation of a portable electrode array for a resistivity fish counter. Fish. Manage. Ecol., 3, 129-141.

SMITH G.W., JOHNSTONE A.D.F., SHEARER W.M., 1996. The behaviour of returning adult Atlantic salmon (Salmo salar L.) at a borland lift fish pass as determined by radiotelemetry. Fisheries Research Services Report No. 7/96. Scottish Office Agriculture Environment and Fisheries Department, $12 \mathrm{p}$.

SMITH G.W., CAMPBELL R.N.B., MACLAINE J.S., 1998. Regurgitation rates of intragastric transmitters by adult Atlantic salmon (Salmo salar L.) during riverine migration. Hydrobiologia, 371/372, 117-121.

STONEHEWER R.O., WALTERS D.W., MEE D.M., 1993. A draft report on the results of the Tawe barrage fisheries impact studies 1993. National Rivers Authority, Welsh Region, South West Area, Report No. PL/EAW/93/6. 
STONEHEWER R.O., KIRKPATRICK A.J., MEE D.M., 1995. The results of the Tave barrage post impoundment fisheries impact studies 1993-1994. Environme tt Agency (Welsh Region), Report No. PL/EAW/95/4, 53 p.

WEBB J., 1989. The movements of adult Atlantic salmon in the River Tay. Scotti.h Fisheries Research Report, 44, $32 \mathrm{p}$. 